\title{
DEVELOPMENTS IN HOSPITAL SERVICE
}

\author{
W. E. Hall, F.C.I.S., F.H.A. \\ Director, Division of Hospital Facilities, King Edward's Hospital Fund for London
}

This article is based on sections of a paper entitled 'The Hospital Service-Today and Tomorrow' and on the introductory address when it was presented on May 19, 1960, at the annual conference of the Chartered Institute of Secretaries.

At a very early stage in planning the rebuilding of a hospital or department it is necessary for the staff who work in that hospital-the medical, nursing, technical and administrative staff-to assist the hospital authority to form an appreciation of the future organization and departmental requirements, for it is upon the basis of this 'client's brief ' that the architect must apply his skill to the creation of a building suited to the special needs. In making this assessment of the nature and extent of the services to be provided some attention must be paid to the many changes taking place-changes in medical and nursing techniques, general routines and procedures, all of which must have an influence on space allocations, the geographical relationship of departments, and the layout, equipment, and staffing of those departments. At the same time it has to be remembered that what the nation can afford in revenue and capital expenditure may well fall short of an ideal solution, but ideal solutions are often short-lived, and the obligation to seek a compromise that makes the best use of the money available may have its compensations.

\section{Revenue Expenditure}

As will be seen from the figures given in Table $A$ the annual net revenue expenditure of the hospital service in England and Wales has increased considerably since the beginning of the National Health Service, almost entirely owing to higher levels of prices and remuneration. It is not necessary to discuss these figures in detail, but as to the future it may be assumed that the cost will rise because of the continuing trend of higher wages and prices. Although one does not contemplate any expansion of the service in terms of accommodation, it is more difficult to forecast whether or not some fall in demand may be expected. It is very probable that treatments will become more complex, intensified and costly, and it would be reasonable to suppose that improvements in standards will absorb most of the savings $\omega$ to be obtained by greater efficiency and the $\rightarrow$ economies gained by replacing old unsuitable $\vec{\oplus}$ buildings by modern structures.

\section{Capital Expenditure}

In $1955-56$ the yearly capital expenditure exceeded fro million for the first time since the beginning of the National Health Service; subsequently, it has increased each year and is expected to amount to $£_{25} 5$ million for the current year (1960-6r) and $f_{31}$ million for 196r-62, nearlo $40 \%$ of which will be absorbed by some $15 \%$ major schemes. It is understood that the preserf intention of the Government is that the capital allocation shall then be increased to $£ 50$ million for each of the next five years.

Large as it may seem, this rate of capital expenditure is not nearly enough to catch up with the arrears dating back to the Second World War or earlier. In 1956 the Guillebaud Committee thought that a desirable target would be $£ 30$ million a year for seven years, but at the British Medical Association annual conference in I959 a resolution was carried urging an expenditure of $£ 75^{\circ}$ million over the next ten years. In the long run it may not be a question of the availability of capital, but of whether there are sufficient architects free to do this work, and of the competing claims of all other projects, outside the National Health Service, for both building staff and materials. There is also the fear that too large a proportion of this money will be devoted to the partial improvement and expansion of buildings which by modern standards are no longer structurally suitable for use as intensivecare hospitals. Capital expenditure of this kind is not nearly so productive as that devoted to new buildings, and its worst feature is that it perpetuates ancient and overcrowded hospitals-one in every five is over 100 years old and two out of every three nearly 70 years old-which are 
TABLe A

Revenue Expenditure of Hospital Authorities (England and Wales)

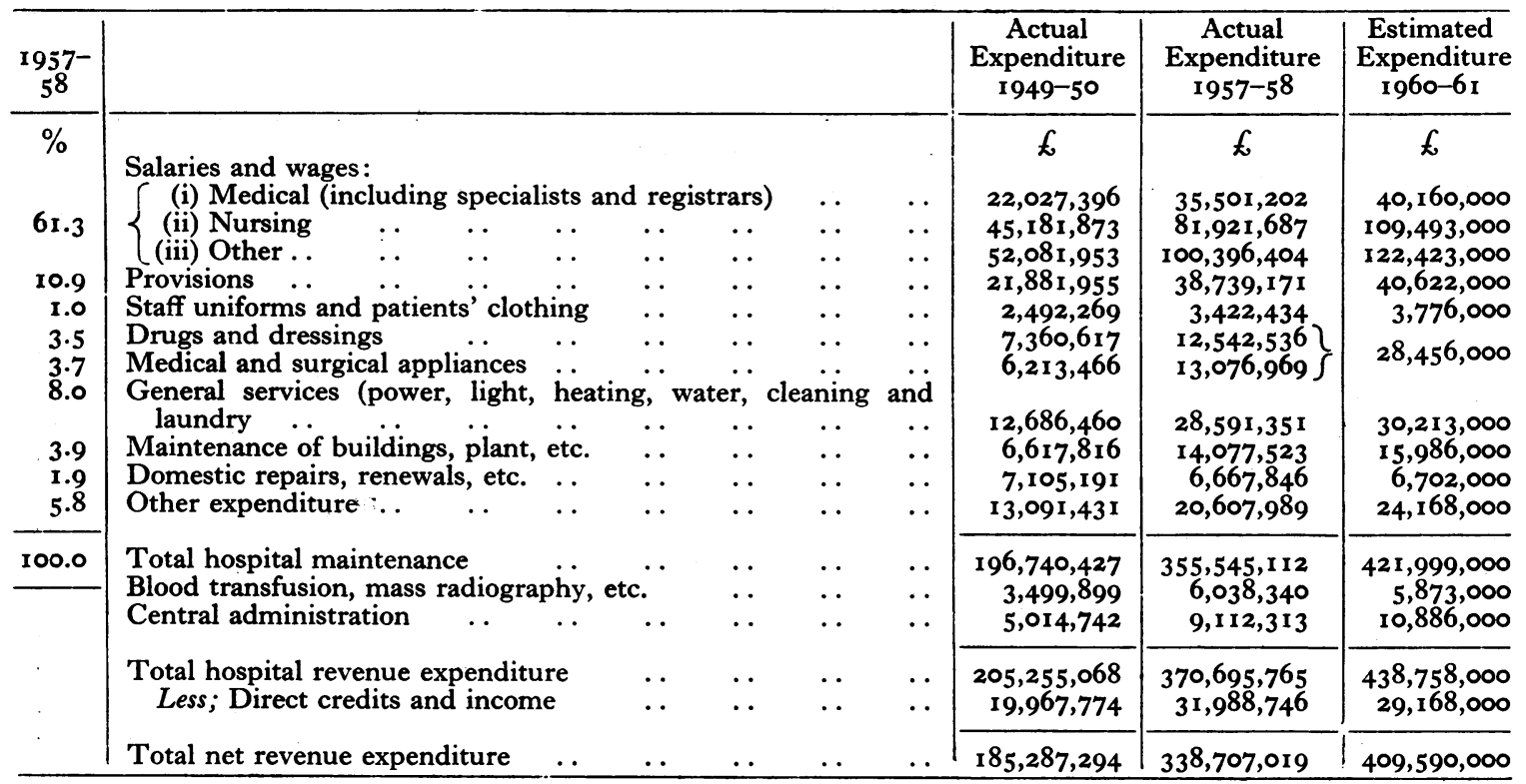

Note.-The above figures have been extracted from the Report of the Ministry of Health for the year ended December 31, 1958, H.M.S.O., Comnd. 806, July, 1959, and from the Civil Estimates, 1960-61, February, 1960. Some of the figures of expenditure and income are not truly comparable with those for other years because of changes in the allocation to headings of certain items of expenditure and income.

extravagant in running costs and offer the most difficult conditions for those who work in them.

It is, of course, of the utmost importance that the national programme for capital development should be designed to the best advantage. One is less concerned with the choice of priorities than with the vital necessity of making sure that the size, location and services of any new building will be in accord with the present and future requirements of the area it serves.

\section{Assessment of Hospital Provision}

Before any hospital is replaced by a new building, or additional hospitals or beds are planned, it is essential to have a guide as to the needs of the population to be served, but the assessment of the hospital provision for a given area is usually hampered by the lack of accurate information and the knowledge that past experience is of little value. The main factors in the equation are (a) population trends, and the influence of industrial, housing and traffic developments, $(b)$ the incidence of disease, $(c)$ advances in medical technique, and $(d)$ the ability to make full use of present and future services.

There has been, for example, a great improvement in the standard of health of children, and a substantial fall in the demand for beds for diseases of the chest, for infectious diseases and for diseases related to poor social conditions, but a considerable increase in traumatic, orthopaedic and neurological surgery. The expectation of life is now much greater. Patients seek treatment earlier and their recovery is quicker; for most specialties the length of stay in hospital is below what it was a few years ago.

The determination of long-term policy in planning hospital provision is one of the main duties of the regional hospital boards, but there is as yet little published information on the extent to which these investigations have been made. Surveys carried out in Northampton, Norwich, Reading and, more recently, in Barrow-inFurness, have confirmed that the figure of 5 or more acute beds for 1,000 population is probably too high, and have indicated that a figure nearer 2.5 acute beds per 1,000 population may be all that will be required in future if the hospital is able to maintain a satisfactory level of occupancy. A significant feature of all these assessments is that the need is estimated to be below the present provision of 3.1 beds per I,000 population, and only about half of what was thought to be the target when the National Health Service came into being.

With a capital cost of approximately $£ 6,000$ 
per acute bed in a non-teaching hospital (it is much more in a teaching hospital), there is good reason to make sure that new hospitals are related to demand, although it does not always follow that the demand must be met in this form. The Barrow-in-Furness survey has shown that, on clinical grounds, a substantial proportion of the beds may be occupied unnecessarily by patients who could have been treated equally well at home. No doubt the greater use of local authority services would help to reduce the expensive occupation of hospital beds.

It should be noted that these estimates of hospital provision assume a reasonably high level of bed occupancy, but it is not easy to maintain a high bed-occupancy rate without some pressure in the demand for beds; and, of course, it is uneconomical to retain patients in acute beds longer than need be merely- because beds are available, in which case the occupancy rate can be misleading. Hospitals seem reluctant to sacrifice one or two beds in a ward to provide space for improvements in structure or services and it is believed that in many instances this could be done without reducing occupied-bed days to any extent.

Some mental hospitals are still overcrowded, but others are beginning to find empty beds, and there is every indication of a substantial fall in the future demand for beds as more and more encouragement is being given to the development of psychiatric centres, out-patient clinics at general hospitals and the establishment of day hospitals. Within the mental hospitals the need is for more occupational therapy, and for greater facilities to enable patients to readjust themselves to normal social life, e.g. by giving them ordinary jobs to do, such as simple ' factory' work.

\section{The Location and Distribution of Hospital Services}

As the result of his studies of the hospital needs of patients in the Birmingham area, Prof. McKeown has advanced strong arguments in favour of a balanced hospital community occupying a common site. In his view, "hospitals should be planned as a group of buildings with a common medical and nursing staff providing complementary services for all classes of patients, rather than as single buildings providing a full range of services for selected groups. The buildings would be of varied size, design and permanence of structure, each closely adapted to the needs of the patient who would occupy them.' The advantages of such a scheme are largely related to improvements in the recruitment, training and distribution of staff, to the flexibility in the use of the buildings, and to the economies arising from the scale of the activities. There is, however, just the danger that patients might feel a little frightened of being lost if the organization $\stackrel{\otimes}{\Omega}$ is too big and all-powerful in the range of services $c$. it provides.

The grouping of hospitals in the National $\stackrel{\vec{F}}{\stackrel{P}{9}}$ Health Service has in many instances provided opportunities to rationalize the work of the group $\overline{\bar{C}}$. by concentrating in one hospital or building the $\frac{\bar{\rho}}{\sigma}$ beds allocated to a given specialty, and, in the $\propto$ case of children's beds and maternity beds in particular, this segregation is regarded as advisable $\vec{\circ}$ on medical grounds.

From some points of view it is perhaps of no $\vec{\omega}$ great consequence whether a particular treatment $\frac{\rho}{\circ}$ service is provided by a department in a general 0 hospital or by a special hospital, provided that the size of the unit warrants the employment of ${ }_{\infty}$ specially trained staff and the installation of the $A$ full range of modern equipment. But other con- $\overrightarrow{0}$ siderations do apply, and, for example, in a densely populated area the concentration of accident ser-

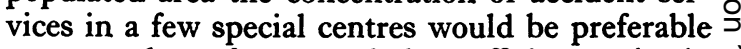
to a number of scattered, less efficient units in $\vec{A}$ which the beds may be filled before the ambulance drivers are aware of it. It is also believed that the mentally handicapped would benefit if, in the 3 course of time, the large institution were replace $\mathbb{\Phi}$ by smaller establishments of more intima character, where patients with similar therapeuti. and social adjustment needs could be brough together.

As part of the long-term policy of building new hospitals, either to originate services in areas of increasing population or to re-establish existing hospital centres, one would like to see, in the early stages, the closing down of most of the structurally and administratively unsatisfactory buildings; at the same time, many of the smaller hospital buildings which are structurally sound, but in design and equipment are too out-of-date to make it worth while to retain them as acute hospitals, might be adapted and maintained at reasonable cost as centres for physiotherapy, radiotherapy, or as hostel-type accommodation.

\section{The Grouping of Patients According to Need 웅}

The above concept of a balanced hospital com- $\frac{7}{0}$ munity made up of a group of special hospital buildings may be contrasted with the traditional $N$ system of mixing patients of different treatment and service needs within the same building, or $N$ even within the same ward.

In America the segregation of patients according to the degree of illness and the need for care is taking the form of 'progressive patient care.' क Under this system the patient is admitted and? transferred, as required, into any of five separate $\frac{T}{0}$ care units, viz. (i) Intensive Care Unit, for patients 
critically or seriously ill, (ii) Intermediate Care Unit, for those who do not require intensive care but are unable to look after themselves, (iii) Self Care Unit, for ambulant and physically selfsufficient patients or those admitted for diagnostic study or preparation, (iv) Long-term Care Unit, for the chronically ill, and (v) Home Care, coordinating medical and related services. These units are not necessarily contained within one hospital, and each will have the facilities and special trained staff to provide the kind of services needed by the group of patients concerned. To the patient paying for his treatment this system has its attractions because the charges will vary according to the services rendered, and in total may be less than what would have been charged previously; but one real objection the patient may have is that, having become familiar with his surroundings, he may then be moved into another building where conditions are changed and there are different staff who do not know very much about him or his idiosyncrasies. Administratively, the system is not without its difficulties, for it will be obvious that fluctuations in bed occupancy between the units could prove to be very wasteful unless flexibility is introduced by making some beds available to one or more of the categories of patients as required.

The idea of grouping hospital patients according to their medical and nursing care needs, and providing accommodation and services to the requirements of those groups, is not new in this country, although there is so far little evidence of it being done other than in a casual or limited degree. It is thought that a simple division between 'acutely ill' and ' recovery' patients would be well suited to the hospital system under the National Health Service and that this may prove to be a more probable form of development than, for example, any extension of the practice of providing 'post-operative recovery' beds. For one reason, compared with the large open ward, the sub-divided ward unit has greater flexibility in the use of beds as between different types of cases, and in these circumstances the separation of the ' acutely ill ' from the ' recovery' cases is not nearly so complicated as it might otherwise be; also, the great advances in medical and surgical technique, chemotherapy, anaesthetics and blood transfusion have speeded up the diagnosis and treatment of the patient so that the acute stage of the illness is both shortened and more readily discernible from the recovery stage, which, too, has become accelerated.

There are two ways in which it is thought this changing situation will be met. In the future planning of wards of acute general hospitals it is believed that more consideration will be given to the designing of a special section of the ward reserved for the seriously ill and post-operative patients. This will be fitted with all services and equipment so that every facility for the immediate care of the patient is near at hand and members of the nursing staff do not have to walk or carry heavy articles for long distances; and being close to the nursing station, the patients will be under constant observation, both day and night, during the critical stages of the illness. Part of the ward will consist of single rooms for the isolation of noisy or infectious patients; the remainder of the ward will consist of open sections of, say, four to twelve beds, for patients who are ambulatory and able to fend for themselves to a large extent, making use of a day room, and taking meals at a table or even visiting a cafeteria within the hospital. In this way the work of the nursing staff of the ward is spread over both light and heavy duties, and the nurses will have the satisfaction of seing the whole of the patient's progress ; it is also true to say that the patients are usually happier if they remain under the care of the same nursing team in the same ward.

An alternative form of this kind of specialization would be to accommodate the two groups of patients in separate buildings, the economical advantage being that in the one building the expensive equipment and services of an acute hospital would be used to the full, whereas in the ' recovery' branch a simpler and cheaper service would be all that was required. Against this undoubted advantage must be weighed the strain on the nursing staff in the acute hospital, the difficulty of equating the numbers of beds in the two buildings so as to maintain the bed occupancy at a reasonable level, and the reaction of transferred patients, who may feel that they are isolated from skilled attention.

\section{The Design of Hospital Wards}

For a very long time the large open ward of some 30 or more beds has been a feature of hospitals in this country, but opinion is now established in favour of the ward unit subdivided into sections, a few containing only one bed and others containing, say, four or six or more beds. A subdivided ward of this kind gives much greater freedom in the use of the beds for different types of cases, but the more sectionalized it is the more difficult it becomes to give adequate nursing attention without adding to the work of the staff or increasing the number of employees. Of course, not all patients like single-bed wards; many prefer to have the company of other patients, and like to see what is going on around them. However, it seems that the pendulum is now swinging back a little to a compromise solution 
which includes both single-bed wards and open sections with ten or twelve or more beds, and such an arrangement would lend itself to the separation of 'acute' and ' recovery' patients as discussed earlier.

Another point is that a subdivided ward unit does add to the area of wall surfaces to be kept clean, but this is offset to an extent by a lower ceiling height of, say, $9 \mathrm{ft}$. or $10 \mathrm{ft}$. compared with $15 \mathrm{ft}$. or so usually found in a large open ward. A lower ceiling height also makes it possible to concentrate more floors in a building of a given height and so to make better use of the cubic space.

The grouping of the beds and the disposition of the service and the ancillary rooms do permit considerable variations in the planning of the ward unit, as can be seen from the innumerable examples which already exist, and it is a subject very susceptible to changing circumstances. In modern hospitals the ward units are often arranged in pairs so that they share the use of service rooms placed between them, such as staff cloak room, relatives' waiting room, medical staff room, etc., thus making better use of the floor space, and the wards are served by the same traffic centres. Separate service lifts for 'clean' articles and 'dirty' articles, carefully sited as part of the overall planning of the ward, would help to reduce unnecessary traffic through the ward, and the patients would benefit from the reduction in noise and general disturbance. It is quite unacceptable that bags of fouled or soiled linen, dirty dressings, food waste and rubbish should be trundled through the wards on trolleys, and it is hardly less so for clean supplies.

The ward sanitary arrangements of many old buildings present an intractable problem. More often than not they are so grossly inadequate that it requires little imagination to outline the improvements one would seek to find in a modern hospital. Certainly the accommodation should be distributed in the ward, rather than concentrated at one end as is often the case at present, and the doorways, fittings, baths, showers and toilet basins so designed as to encourage patients to help themselves and to allow the free movement of wheeled chairs. It is a fact that much time and space are taken up in the ward by equipment which is there for no other reason than that built-in services are not readily to hand.

In American hospitals the utility rooms and cupboards, toilets, plumbing, service ducts and lifts are sometimes placed in the middle of the ward so that as much as possible of the floor area close to the exterior wall, with its fresh air, sunlight and scenery, is then available for the benefit of the patients. Hitherto, this form of layout has not been acceptable in this country, but with adequate mechanical ventilation there is now no reason why it should not become a feature of future planning.

A subject that needs careful thought is the optimum size of an acute ward in relation to thes? ward nursing team. With the intensification ofo treatment services and the shorter length of stayo and turn-over interval, the pressure of work on $\frac{\bar{c}}{\mathrm{~s}}$ the staff may be such that a ward unit of smaller size, say, 20 to 24 beds, may be an importanto factor in obtaining the greatest effective use of ${ }^{\text {s }}$ hospital beds, although, as mentioned above, two. such units could be linked together to share certain $\vec{\omega}$ common services.

There are, of course, many other ways in: which the ward of the future may differ from that 3 . of the past and a number of these changes havew already taken place, sometimes in experimentali: form. Examples are given elsewhere in this $\overrightarrow{0}$ article, but to quote others: Built-in storage for patients' clothes, which together with the over-o bed table may lead to the conversion of the rathero cumbersome bedside locker into a small cabinet hinged from the wall incorporating the reading $\infty$ light, wireless and two-way communication con- $\frac{0}{0}$ trols; special door furniture, with silent locks; 3 windows, double-glazed to reduce noise and heyt loss, that are not too large because excessive suglight can distress patients and sunblinds are then necessary; windows that can be swivelled $\bar{\oplus} 0$. permit easy cleaning from inside the building but can be locked to limit movement for reasons of safety; equipment reserved for the exclusive $\bar{\circ}$ use of the individual patient, and subject to terminal sterilization on his discharge and before $\stackrel{\unrhd}{\circledR}$ it is re-issued; flush doors, coved skirting, $\overrightarrow{\overrightarrow{0}}$ radiussed corners, and the absence of unnecessary 3 ledges to prevent the collection of dirt; cubicle? curtains of glass fibre, plastic, treated cotton, etc., , which discourage the penetration of dirt and can be washed and drip-dried very quickly; local 3 . incineration for the immediate destruction of 0 dirty dressings; a comfortable day-room where patients can obtain a change of environment or,, in the case of a geriatric ward, where occupational $₹$ therapy and remedial exercises may be practised.음

\section{Out-Patient and Casualty Departments}

Each year there are approximately 28 million out-patient attendances for treatment or consulta- tions, these patients having been referred to the $N$ hospitals by their general practitioners. Today, N nearly all hospitals arrange for their patients to come by appointment to ensure that, so far as cano be reasonably arranged in the circumstances, the time spent at hospital is as short as possible and $\stackrel{?}{+}$ that the hospital services are used economically. 뭉 
In modern hospitals the very large crowded and noisy waiting hall has been replaced by small, comfortably furnished clinic waiting areas, with the dressing, examination and consultation rooms designed to meet the special needs of the individual clinics. The patients' cafeteria is another amenity.

In addition, there are approximately I I million attendances at casualty departments. Of the 6 million or so patients attending these 'accident, emergency and dressing stations,' it is thought that about one-third would be suffering from more serious conditions such as multiple injuries, burns, scalds, fractures, etc., which need immediate and accurate diagnosis and intensive treatment. With increasing mechanization and traffic the number and severity of injuries are growing, and once again attention is being given to the design of a fully comprehensive national accident service, with centres specially equipped and staffed for the task.

There is also reason to believe that the number of patients admitted into hospital could be greatly reduced, and the length of stay shortened, if the diagnostic and investigation facilities in outpatient departments were expanded and the hospital and general practitioner services were more closely related. If a patient can be treated just as satisfactorily at home, the cost of doing so might be no more than one-quarter of the cost of keeping that patient in an acute bed.

On the other hand, if the investigations on admission are properly organized the financial savings to be obtained by pre-admission investigations in the out-patient department may not be substantial, and the comfort of the patient must also be taken into account.

\section{Catering}

It is accepted that good food, properly cooked and attractively served, makes an important contribution to the treatment, well-being and recovery of every patient, and it is no less important that hospital staff should have a high standard of feeding, particularly residential staff who will depend upon the hospital for an adequate and varied dietary. Expenditure on provisions alone exceeds $£ 40$ million a year, and to this must be added the cost of the ordering, storage, preparation, cooking and service of the food.

During the past ten years there have been considerable improvements in hospital catering, both in nutritional standards and in presentation, but unfortunately no more than $2.5 \%$ of the limited capital expenditure has been devoted to structural improvements to main kitchens. In many old hospital buildings the unsatisfactory layout of the kitchens and the poor equipment add greatly to the difficulties of providing three main meals daily to, say, some 2,000 persons at approximately the same times, apart from which there is the further problem of distributing the hot food to the wards, in some instances in ward blocks not equipped with lifts. Decentralization of the work into smaller kitchens can sometimes bring about an improvement in service in such circumstances, and may even be more economical.

So far as the design of hospital kitchens of the future is concerned, it seems probable that more attention will be paid to compact planning, air cooling and ventilation, quietness, refrigeration (including garbage), and to the extended use of stainless steel and mobile equipment so as to facilitate cleaning. In new buildings of suitable design it is possible that moving belts and lifts may be installed to provide a direct tray service to the wards and for the return of dirty crockery to the central wash-up. A cafeteria service for patients is another feature likely to be developed.

The maintenance of a good standard of hospital catering is also dependent upon the efficiency of the catering staff, and when national salary scales tend to be low in comparison with local commercial levels it becomes hard to attract and retain skilled staff, especially the fully qualified and experienced men and women needed as heads of hospital catering departments. Notwithstanding the present shortage of qualified dietitians, it is to be hoped that in future more hospitals will be able to appoint dietitians; by modern standards the medical services of a general hospital are not complete without the assistance of a dietitian in the preparation of special diets, and in demonstrating dietary practice to patients and student nurses.

\section{Planning and Building New Hospitals}

Because of the time it takes to plan and build a hospital, and the continuous rapid advances in medical knowledge and technique, a hospital may soon be out of date. It is therefore reasonable to include in the original design some provision for a limited flexibility in the space allocations, and even for modest expansion, but a hospital which is out of balance because parts of it are disproportionately expanded, or because it remains uncompleted for a number of years, may prove to be uneconomical in maintenance costs.

It is claimed that, compared with a multistorey building, a single-storey structure is cheaper and easier to build, allows more freedom for the future growth of departments, and reduces the time absorbed in traffic movements within the building. On the other hand, in a multi-storey building the pattern of a ward, for example, can be repeated vertically; this reduces the cost of plumbing and other expensive services, gives 
opportunities for mechanical transport by lifts, and offers considerable possibilities of savings in cleaning, heating and lighting costs because of the compactness of the building and the reduction of the space taken up by corridors. One may also doubt whether it is good policy to use up valuable land by a sprawling single-storey building; surely it is preferable to see a small area taken up by a compact building and the remainder of the site developed as recreational gardens, isolating the hospital from the noise of traffic, etc.?

The size of a hospital is obviously a matter of great importance, for at one end of the scale there is the hospital which, although providing essential services, is too small to be economical in maintenance, whereas at the other end there is the very large hospital which tends to be unwieldy to manage and unfriendly in its general atmosphere. The indications are that for a general hospital of the future a range of 400 to 600 beds might be regarded as an optimum size both from the point of view of management and staffing as well as the need for each of the constituent departments to be large enough to provide an economical service. Additional considerations apply in the case of a teaching hospital. Here it is necessary to relate the patient services to the teaching facilities, and, in order to include a large range of departments, each of appropriate size, it would seem that a total of some 900 to $\mathrm{r}, 000$ beds would be required, though not necessarily all on one site.

\section{Hospital Design}

By its very nature, in hospital design there will always be room for experiment and differences of opinion, and, having regard to the slow rate of future building even in the most optimistic circumstances, it is to be hoped that individual architects and hospital authorities will be allowed the utmost freedom in planning and developing new ideas. Once built, hospitals are likely to be with us for a very long time, and it would be very sad if those to be constructed during the next decade or so were to follow any centrally dictated concept of shape and layout. Of even greater importance is the need to avoid any inhibitions in the choice of finishing materials imposed by a conscious limitation in the amount of capital money to be made available for the project. Because the Treasury accounts are kept on a cash basis there is a far too pressing tendency to control today's spending without regard to tomorrow, so that it becomes necessary to emphasize the wisdom of relating capital expenditure to the constantly recurring maintenance expenditure arising from it.

Within the compass of this article it is not possible to discuss at length all the features which make up the design of a modern hospital In this field a good deal has been learned fromb the extensive hospital building programmes which have been proceeding rapidly in all parts of the world, and in other ways-for example, the build ing of schools-where experience has been gainedo in the use of new materials and structural formso as well as in the management of construction $\overline{\bar{c}}$. programmes so as to save time and money. Ship- $\frac{\mathbb{D}}{2}$ building also offers some most valuable lessonso in the conservation of space and in the design of built-in fittings that could be applied to many of the problems of hospital planning. One way in $\vec{\omega}$ which hospitals of the future will differ frome those of the past is that the new buildings willo be much more compact. The reservation of specially designed accommodation for the exclusive part-time use of individual departments cani be wasteful and, instead, architectural skill mayç now be more concerned with designing accom- $-\mathrm{H}$ modation for a variety of uses and for occupation for a greater proportion of available time.

Unnecessary noise can be both irritating andtiring to all persons, and even more so to the sick ${ }_{\mathbb{D}}$ patient, whose recovery may be retarded. Most of the complaints from patients refer to high- -3 pitched or intermittent sounds, which are not? necessarily loud, but are often caused by equipisment which has not been maintained in goedso condition or by the lack of thought on the pait of individuals, and so the remedies are near to hand. So far as the hospitals of tomorrow ares concerned, it seems likely that questions of noise will have an increasing influence on structural design, departmental layout and finishing ma- $\stackrel{\mathbb{Q}}{\stackrel{2}{2}}$ terials, but in many matters there will probably $\overrightarrow{\overrightarrow{0}}$ remain a conflict of choice in which the require- $\exists$ ments of hygiene must be given preference.

From a functional point of view, three of theo most powerful influences affecting the design of the future hospitals are $(a)$ the increasing ten- 3 . dency towards the centralization of certain ser-o vices, $(b)$ closer attention to reducing the risks of cross-infection, and $(c)$ the need to conserveo manpower.

\section{Centralized Services}

Examples of the present trend towards cen-을 tralization are the washing and sterilization of crockery, staff cafeteria, central sterile supplyo services, central linen services, and the preliminary washing of fouled linen by centralized machinery N so as to avoid the sluicing of these articles in the ward areas. Considerable savings have beeno obtained by the centralization of laundry work, particularly when the volume justifies the use of automation and specialized machinery, but there 
are time and cost limits to the distribution area that may be covered economically. The following are further examples of centralization, in different forms, viz. pneumatic-tube systems for the conveyance of mail, drugs, X-ray films, etc.; piped supply lines for the distribution of oxygen, compressed air, distilled water, medical suction; magnetic induction and wireless call systems for staff location and the emergency control of apparatus; electronic and mechanized equipment for centralized accounting; central dictationrecording apparatus, linked with the internal telephone system, for recording doctors' correspondence and medical notes; centralization of medical records and admission services, etc.

The establishment of a central sterile supply department within the hospital makes it possible to employ modern equipment in the care of specially trained staff, thereby obtaining a greatly improved standard of sterilizing procedure. In the last few years the demand for apparatus of higher technical efficiency has led to great advances in the design of autoclaves, including highspeed high-vacuum models, and more reliable methods of using ethylene-oxide sterilization, as well as the introduction of new methods, such as the cleaning of surgical instruments by ultrasonic equipment. In many hospitals drum sterilization has been replaced by package sterilization, using calico, muslin, cardboard boxes, nylon film or paper as inner or outer wrappers, arranged in such a way that the outer wrapper forms the sterile field; it would be of advantage to hospitals generally if there could be a measure of standardization in the range and contents of these packages.

A development which is likely to have a profound influence on hospital practice is the use of gamma radiation as a sterilizing medium. The outstanding characteristic of this method is that it is possible to sterilize goods previously packed in an air-tight bacteria-proof container, and its application to the manufacture of surgical dressings and disposable items could lead to considerable economies in hospital procedures. Disposable catheters, sterilized in this way, are already being supplied to hospitals, and the method may well suit the cheaper plastic materials which will not stand up to autoclaving temperatures, but it remains to be seen whether or not a competitive processing cost is possible and can be maintained. No hospital is as yet equipped with a gamma radiation unit because of the very high cost of an experimental unit.

In future the possibilities are that disposable needles, syringes and other items of equipment will be more widely used, and when these are perfected and produced in mass it may well be that the cost will be less than that of the cleaning, re-sterilizing, safe storage and issue of standard equipment; this is already so in the case of surgical needles.

\section{Reducing the Risks of Cross-infection}

A serious problem to hospitals at the present time is that of cross-infection due to antibioticresistant staphylococci. Some of the preventive measures to be taken have a bearing on planning or general procedures as distinct from medical or nursing techniques, and reference has already been made to the inclusion in the ward unit of a number of single rooms for the infected patients or the temporary isolation of new patients.

It is accepted that dust plays an important part in the dispersal of organisms, and the problem is one of keeping the volume and movement of dust to the minimum without impairing the ventilation. In operating theatre suites, where the humidity and temperature of the air are as important as its cleanliness, it is now the practice to install positive pressure air-conditioning plant, but this is too expensive for other departments of the hospital, desirable though it may be. For the wards and treatment rooms it is at least possible to see that fresh air is supplied through filtered louvres and to control the movement of air by fans, and we may expect to see this done in examples of future planning.

The sweeping of wards by brooms has long been viewed with disfavour, and no doubt vacuum cleaners would have become more widely used had they not been suspected of acting as distributors of finely divided, and possibly infected, dust passing through the collecting bags, and of disturbing dust by the exhaust air. Modifications have now greatly improved the dustcollecting efficiency of these machines and the method of disposing of the collected dust, but in new buildings built-in piped suction cleaning may be preferred as being more effective in action, simpler and quieter to use. Now that it is possible to lay a floor covering with welded joints, the cleaning of ward floors by a wet or modified 'flooding' process is being examined with a view to including suitable disinfectants in the cleaning fluid, and using suction-drying machines.

The recognition of the hospital blanket as a potential reservoir of infection has led to greater interest being taken in the cleansing and sterilization of blankets of wool, cotton and other materials. The search is for more effective bactericidal agents and laundry procedures which will enable blankets of various types to be washed much more frequently than hitherto without deterioration, and for better standards of hygiene. The process must also be simple, otherwise some 
TABLE B

Examples of Costing Statistics Relating to Hospitals Operating the Main Scheme, for the Year Endep⿱ MARCH 31, 1959 (ENGLAND AND WaLes)

(I) IN-PATIENT DEPARTMENTS (WARDS)

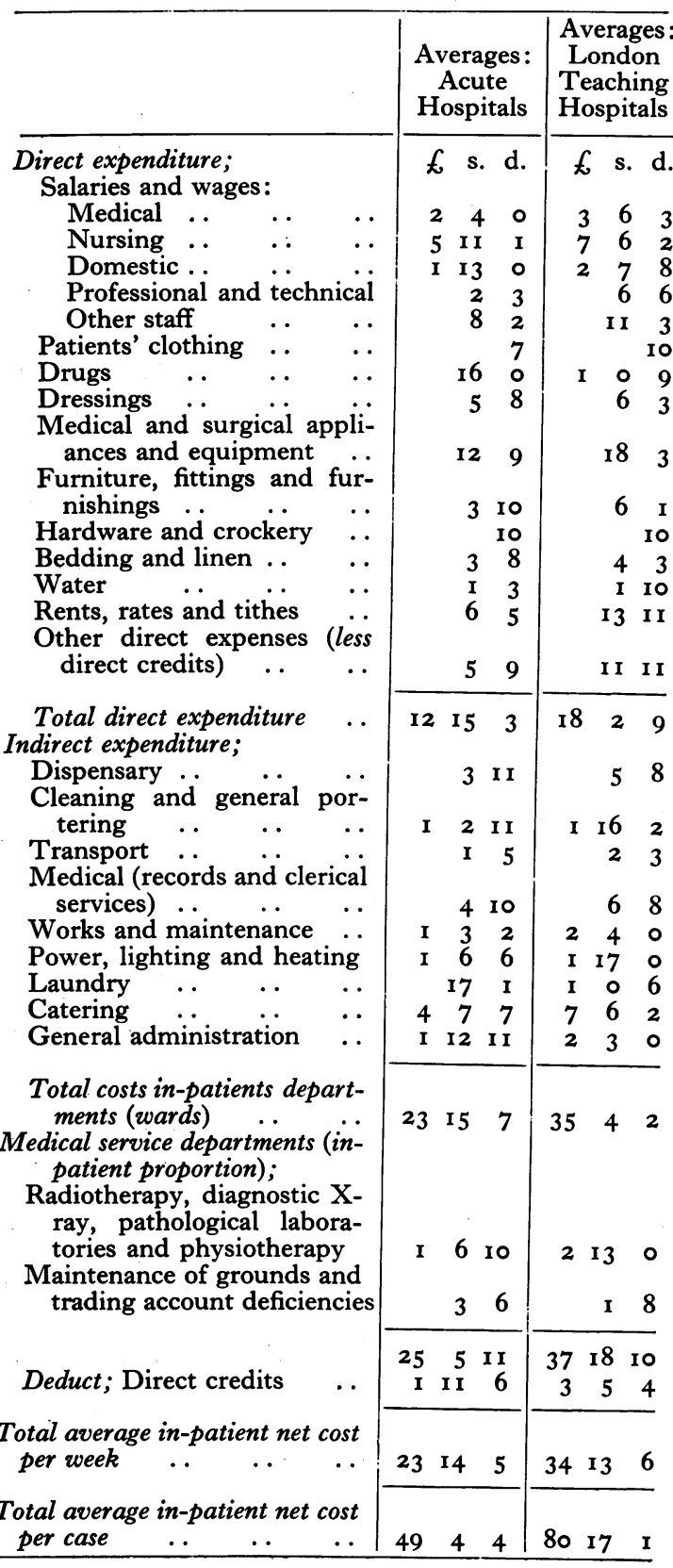

hospital laundries may have difficulty in meeting the greater volume of work that has now arisen. Attention is also being given to the wrapping of clean linen so that it may be returned from the laundry and stored with the minimum risk of contamination.
(2) EXAMPLES OF DEPARTMENTAL UNIT COSTS

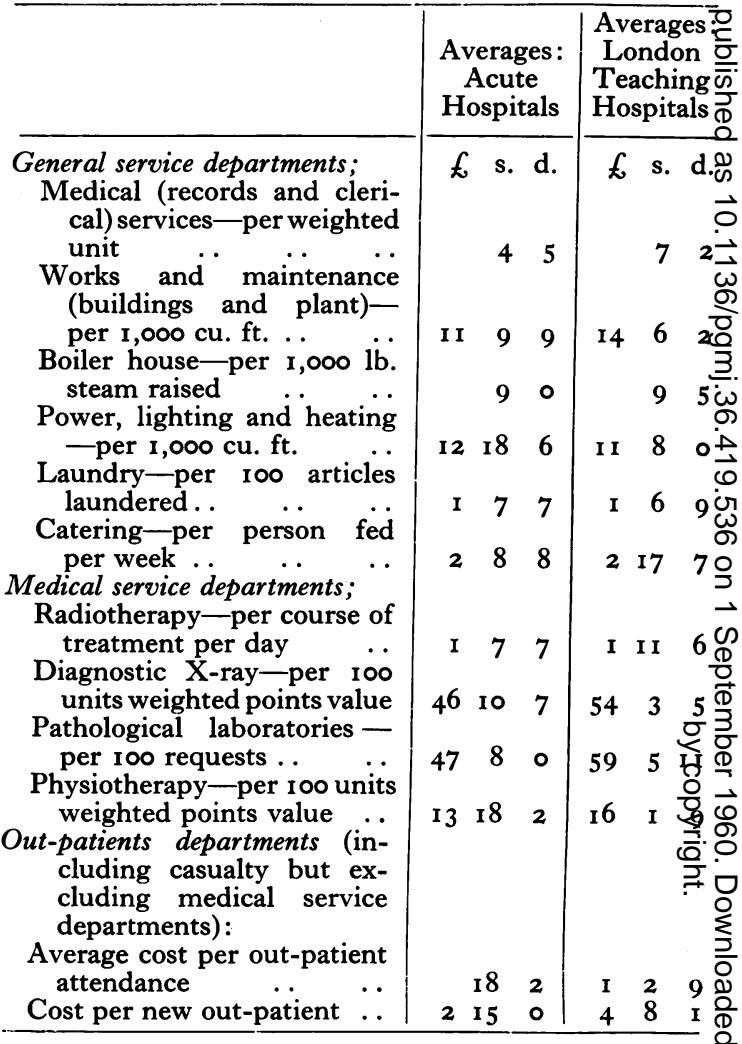

Note.-The above figures are extracted from the $\overrightarrow{\overrightarrow{0}}$ National Health Service Hospital Costing Returns for 3 the year ended March 3I, 1959. H.M.S.O. The main scheme of costing provides for the departmental accounts to include the expense of services rendered to them by other departments in addition to their own direct expenses. The in-patient account summarizes the whole of the expenditure other than that upon outpatients, and expresses the result as unit costs-viz., cost per in-patient week and cost per case.

New plastic materials promise to replace rubber sheeting, and new types of mattresses made of plastic foam and plastic mattress covers are undergoing hospital tests; as in the case of $N$ terylene-filled pillows and plastic foam pillows, $N$ these articles have the advantage that they can $N$ be autoclaved. Another proposal is to establish within the hospital a 'bed exchange centre,' as has been done in Holland and America. This will issue a clean bedstead complete with bedding on the admission of a patient and receive the returned equipment on the patient's discharge, an arrangement which will also facilitate ward 
cleaning because there will be no empty beds, only empty bed spaces.

The main advantages of disposable items are that they greatly reduce the risks of cross-infection and, in some cases, provide a more acceptable way of dealing with unpleasant tasks. They save the cost and the care that must be taken in the cleaning, sterilization and transportation of nondisposable equipment, but against this must be taken into account the purchase price of the articles and the cost of storage, issue and disposal. The range of paper items continues to expand: for example, paper crockery, containers for medicines and food, paper towels and tissues, table mats, bath mats, face masks; bags for refuse collection, for soiled and fouled linen, and for dirty dressings; sputum containers, bed-pan covers, incontinence pads, etc. Paper sheets for beds have been used experimentally, also paper gowns for patients being X-rayed and for staff in the operating theatre. Disposable items are also made of other materials, e.g. plastic beakers, petri-dishes and mittens, and disposable rubber gloves and catheters. In some instances the costs are not economically attractive, although this situation may change if large-scale production can be encouraged.

There is no doubt that many of our hospitals are not nearly as clean as they should be, and closer attention to normal standards of hygiene would itself do much to help the battle against cross-infection. This concerns both the routine day-to-day cleaning as well as the more thorough periodical cleaning which includes washing-down and re-decoration. The greater part of the latter work is usually done while the wards are occupied with patients, a practice which may now be called into question. A better arrangement, already being adopted by some hospitals, is to have a 'transfer' ward so as to enable each ward to be closed in turn for thorough cleaning at regular intervals, thus making it easier to plan in advance a well-balanced, integrated programme of structural maintenance and domestic hygiene.

\section{Conservation of Manpower}

The position of corridors, the adequacy and siting of lifts, the geographical relationship between departments, all have a bearing on the time and energy absorbed in the movement of persons and goods, and of even greater consequence is the planning of the layout of wards and departments so that facilities are close at hand. Unfortunately the effects of bad planning can be accumulative, for the solution then often takes the form of additional equipment which brings with it its own problems of storage, transportation and maintenance. To give other examples: Laminated and sheet plastic materials for working surfaces and decorative finishes can play a great part in reducing the cost of cleaning and renewal of paintwork, items of expenditure which are heavy in labour cost. From trials now taking place it is already clear that for some flooring materials a durable non-slippery surface can be more easily and quickly obtained with floor sealers and emulsion polishes than with other forms of floor maintenance, this reducing the expenditure on wages. A central linen service saves all the wasted effort in marking, checking and sorting linen according to wards and departments, and by treating clean linen as a consumable commodity' instead of ' ward inventory items' it is possible to dispense with all linencounting in the wards. Automation is being applied to a number of procedures, such as the control of the washing machines in the laundry, automatic X-ray film processing units, and automatic analysis and staining equipment for routine laboratory investigations. A central stores building planned for that purpose and fitted with modern storage equipment and mechanical handling apparatus can save space, unnecessary movement and checking. The simplication of clerical procedures is yet another example.

Manpower is the most expensive item in the running cost of hospitals, and there is every likelihood that the salaries and wages bill will tend to become higher as rates are increased in competition with industry, where the employees expect to share in the results of greater productivity. Within such a personal service, it is, of course, difficult enough to reduce manpowermore so when buildings and equipment are so out of date-and it is therefore of the utmost importance to ensure that in designing hospitals of the future every attention should be paid to conserving manpower. 